\title{
Industrial-Scale Study of the Chemical Composition of Olive Oil Process-Derived Matrices
}

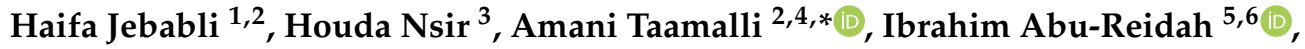 \\ Francisco Javier Álvarez-Martínez ${ }^{7}$ (), Maria Losada-Echeberria ${ }^{7}$, Enrique Barrajón Catalán ${ }^{7}$ \\ and Ridha Mhamdi ${ }^{2}$ (D) \\ 1 Faculty of Mathematical, Physical and Natural Sciences of Tunis, University of Tunis El Manar, Tunis 2092, \\ Tunisia; jebebli@hotmail.com \\ 2 Laboratory of Olive Biotechnology, Center of Biotechnology of Borj-Cedria, P.O. Box 901, Hammam-Lif 2050, \\ Tunisia; ridha.mhamdi@cbbc.rnrt.tn \\ 3 Mediterranean Institute of Technology (Medtech), South Mediterranean University, \\ Les Berges du Lac II 1053, Tunisia; houda.nsir@gmail.com \\ 4 Department of Chemistry, College of Sciences, University of Hafr Al Batin, P.O. Box 1803, \\ Hafr Al Batin 39524, Saudi Arabia \\ 5 Industrial Chemistry Department, Faculty of Sciences, Arab American University, P.O. Box 240, \\ Zababdeh-Jenin 13, Palestine; iabureidah@yahoo.com \\ 6 Department of Environmental Science/Boreal Ecosystem Research Initiative, Memorial University of \\ Newfoundland, 20 University Drive, Corner Brook, NL A2H 5G4, Canada \\ 7 Instituto de Biología Molecular y Celular (IBMC) and Instituto de Investigación, Desarrollo e Innovación en \\ Biotecnología Sanitaria de Elche (IDiBE), Universidad Miguel Hernández (UMH), 03202 Elche, Spain; \\ f.alvarez@umh.es (F.J.Á.-M.); mlosada@umh.es (M.L.-E.); e.barrajon@umh.es (E.B.C.) \\ * Correspondence: ataamalli@uhb.edu.sa
}

Received: 2 May 2020; Accepted: 15 June 2020; Published: 17 June 2020

\begin{abstract}
The effect of the industrial process and collecting period on produced olive oil and by-products was evaluated. Obtained results showed significant variations for the majority of quality indices before and after vertical centrifugation between all samples from the three collecting periods. All samples were rich in monounsaturated fatty acid: Oleic acid (C18:1) with a maximum of $69.95 \%$. The total polyphenols and individual phenolic compounds varied significantly through the extraction process, with a significant variation between olive oil and by-products. Notably, the percentage of secoiridoids and their derivatives was significant in paste and olive oil, highlighting the activity of many enzymes released during the different extraction steps. Regarding antioxidant capacity, the most remarkable result was detected in olive oil and olive mill wastewater samples.
\end{abstract}

Keywords: olive oil; olive paste; by-product; industrial process; phenolic compounds

\section{Introduction}

Olive oil represents the main ingredient of the traditional Mediterranean diet, thanks to its numerous beneficial effects on human health. The health benefits attributed to this product are mainly due to its richness in antioxidants [1]. Indeed, many scientific studies confirm that antioxidant compounds (tocopherols and polyphenols) are responsible for the reduction of the risk of coronary disease and degenerative diseases such as atherosclerosis, cancer, and strokes [2]. On the other hand, olive oil production represents an essential agro-industrial activity in the economic sector of many Mediterranean countries. Currently, the production of olive oils uses the continuous two-and threephase processing systems because of their higher capacity, shorter processing, and reduced storage time and workforce costs [3]. This sector results in a high production of waste that can reach 30 million 
tons per year [4]. Olive oil extraction generates two main by-products: A solid residue (pomace) and an effluent known as olive mill wastewater (omww) [5]. Pomace represents a raw material for the extraction of pomace oil and is also used as fuel and for generation of biomass by microbiological processes. Olive mill wastewater has no economic value, representing a worthless by-product of the extra virgin olive oil industry and an additional cost for disposal pretreatment [6]. Such by-products are responsible for severe environmental problems because of their high concentration in organic acid that turns them into phototoxic materials [7]. Particularly, omww has a substantial level of polyphenols that have a toxic effect on individual plants and microorganisms [8]. However, these by-products may represent a promising source of bioactive molecules [9]. Several researchers have reviewed literature data concerning the composition of olive oil by-products in terms of phenolic compounds and have highlighted interesting results regarding the antioxidant and biological activity of phenolic extracts from wastewater and pomace [10]. As an approach to reduce the negative impact of olive oil industry by-products, the phenolic compounds derived from olive oils and by-products are now used in food, cosmetic, and pharmaceutical industries. Olive by-products show a high concentration in secoiridoid derivatives [11].

Interestingly, omww has high levels of phenolic compounds, which could be transformed into a natural source of valuable and powerful antioxidants [12]. Additionally, the phenolic compounds present in the omww have registered a potent biological activity. In particular, hydroxytyrosol is considered a protective agent of blood lipids against oxidation according to the European Food Safety Authority $[12,13]$. According to literature, the phenolic compounds present in the dry olive residues have antiproliferative activities against breast cancer [14]. Regarding olive pomace, it represents a high potential in the production of a functional food because of its low-cost and richness in phenolic compounds. In fact, the anti-inflammatory effect of olive pomace extract has been clearly demonstrated where the polyphenols have expressed a high therapeutic potential in intestinal bowel disease [15].

The principal aim of this work is to screen the quality of some olive oils produced in Tejerouine (Kef region, in the north-west of Tunisia) and to study the effect of production process on the chemical composition of olive products and by-products using a three-phase industrial scale system for three harvesting dates.

\section{Materials and Methods}

\subsection{Sampling}

All samples (paste, olive oil, omww, and pomace) were obtained from a three-phase continuous chain olive mill in Tejerouin in the Kef region (north-west of Tunisia). The collected samples come from the following cultivars: Sample one: Chetoui, samples two, three, five, and seven: Chetoui and Chemlali, sample four: Chetoui and Gerboui, and sample six: Chetoui and Koroneiki. In blended samples, the dominant cultivar was Chetoui which is the autochthonous cultivar of the Kef region. Sampling was carried out in the beginning (d1: 22/11/2017), middle (d2: 19/12/2017), and the end (d3: 19/01/2018) of the crop's season. From the non-centrifuged oil, omww and pomace were obtained after horizontal decantation step. From the centrifuged oil and omww were collected after the vertical centrifugation step.

For each sample, the same orchard and the same percentage of the different cultivars in the blend were considered for the three collecting dates.

\subsection{Solvent-Extraction of the Polar Fraction}

\subsubsection{Extraction of Polar Fraction from omww}

Two methods were used for the extraction of the polar fraction from omww.

Method (1) was as described in literature [16] with some modifications. The omww mixed with hexane was agitated and centrifuged. Then the delipidated omww (aqueous phase) was collected 
for the liquid-liquid extraction. At this point, ethyl acetate was added (v/v). After agitation and centrifugation, two phases were obtained representing a supernatant, rich in polyphenols, and a base, which was omww. The recovered organic phase was evaporated under vacuum in a rotary evaporator at $38^{\circ} \mathrm{C}$. The obtained residue was dissolved in methanol for further analysis.

Method (2) consisted of evaporating $8.33 \mathrm{~mL}$ of omww under vacuum in a rotary evaporator at $40{ }^{\circ} \mathrm{C}$. The residue was mixed with methanol/water then dried by rotary evaporator at $40{ }^{\circ} \mathrm{C}$, then methanol was added to the residue before analysis.

\subsubsection{Extraction of Polar Fraction from Olive Pomace and Paste}

Phenolic extracts from dry pomace and paste were obtained by the conventional method described in the literature [17]. Briefly, $1 \mathrm{~g}$ of dry matter was dissolved in $10 \mathrm{~mL}$ methanol. The samples were maintained for $24 \mathrm{~h}$ in the dark under agitation at room temperature. The extract was then filtered and evaporated under vacuum in a rotary evaporator at $38^{\circ} \mathrm{C}$.

\subsubsection{Extraction of Polar Fraction from Virgin Olive Oil Samples}

The phenolic extract of olive oil was obtained as follows: $2.5 \mathrm{~g}$ of olive oil was dissolved in $5 \mathrm{~mL}$ of hexane. Subsequently a mixture of methanol:water $(60: 40, \mathrm{v} / \mathrm{v})$ was added. After that, the mixture was agitated and centrifuged at $3500 \mathrm{rpm}$ for $10 \mathrm{~min}$. The polar phase, was recovered for analysis.

\subsection{Quality Indices}

Determination of free fatty acids (FFAs) given as \% of oleic acid, peroxide value (PV), absorbance in the UV $\left(\mathrm{K}_{232}\right.$ and $\left.\mathrm{K}_{270}\right)$ was carried out according to the standard methods described by the International Olive Council (IOC) [18]. The free acidity was determined by titration of a solution of oil dissolved in ethanol/ether (1:1) with $0.1 \mathrm{M}$ potassium hydroxide in ethanol. Peroxide content was established by the reaction of a mixture of $1 \mathrm{~g}$ of oil and chloroform/acetic acid with a solution of potassium iodide in darkness. The free iodine was then titrated with a sodium thiosulfate solution. PV was expressed as milliequivalents of active oxygen per kilogram (meq $\mathrm{O}_{2} \mathrm{~kg}^{-1}$ ). $\mathrm{K}_{270}$ and $\mathrm{K}_{232}$ extinction coefficients were calculated from the absorbance at 270 and $232 \mathrm{~nm}$, respectively, with a UV spectrophotometer using a $0.1 \mathrm{~g}$ of olive oil in cyclohexane and a path length of $1 \mathrm{~cm}$.

\subsection{Pigment Content}

Chlorophylls and carotenoids were determined colorimetrically. The absorbance at $670 \mathrm{~nm}$ was specific to the chlorophyll fraction, and that at $470 \mathrm{~nm}$ was specific to carotenoids. The applied values of the specific extinction coefficients were $\mathrm{E} 1=613$ for pheophytin as a major component in the chlorophyll fraction, and E2 = 2000 for lutein as a major component in the carotenoid fraction. The pigment contents were calculated as follows:

$$
\begin{aligned}
& \text { Chlorophyll }(\mathrm{mg} / \mathrm{kg})=\left(\mathrm{A} 670 \times 10^{6}\right) /(\mathrm{E} 1 \times 100 \times \mathrm{d}) \\
& \beta \text {-carotene content }=\left(\mathrm{A} 470 \times 10^{6}\right) /(\mathrm{E} 2 \times 100 \times \mathrm{d})
\end{aligned}
$$

E1: The specific extinction for pheophetin

E2: The specific extinction for lutein

where, $\mathrm{A}$ is the absorbance and d denotes the spectrophotometer cell thickness $(1 \mathrm{~cm})$.

\subsection{Trolox Equivalent Antioxidant Capacity (TEAC)}

TEAC was performed by preparing a solution of 2,2'-azinobis (3-ethylbenzothiazoline 6-sulfonate (ABTS) in distilled water and a solution of potassium persulfate. After that, in a 96-well plate, $200 \mu \mathrm{L}$ of ABTS was added in all the well plate then $20 \mu \mathrm{L}$ trolox with different dilutions already prepared, and 
$20 \mu \mathrm{L}$ of extract were added to the first line well plate. Measures were finally performed using a plate reader. TEAC was expressed as mmol of trolox equivalent (TE) per $100 \mathrm{~g}$ of dry weight of extract (d.w.).

\subsection{Determination of Total Polyphenols}

The total polyphenol contents were determined in each extract using Folin-Ciocalteu's method adapted to a 96-well plate assay and expressed as percent of gallic acid equivalent (\% GAE per extract) [19]. A calibration curve of gallic acid was prepared. Then, $10 \mu \mathrm{L}$ of the extract was mixed with $50 \mu \mathrm{L}$ of Folin-Ciocalteu. Then after addition of $100 \mu \mathrm{L}$ of sodium carbonate $\left(\mathrm{Na}_{2} \mathrm{CO}_{3}\right), 840 \mu \mathrm{L}$ of distilled water was added. After $20 \mathrm{~min}$ in the dark. The reading was accomplished at $700 \mathrm{~nm}$.

\subsection{HPLC-MS Analysis of the Polar Fraction}

The polar fractions of the paste, olive oil, and by-products were analyzed using high-performance liquid chromatography coupled to mass spectrometry (HPLC-MS). An analytical technique that combines the physical separation capabilities of liquid chromatography and the mass analysis capabilities [20]. The phenolic compounds of the different extracts were separated using Agilent LC 1100 series (Agilent Technologies, Inc., Palo Alto, CA, USA) controlled by the Chemstation software and equipped with a pump, autosampler, column, and UV-Vis diode array detector. The HPLC instrument was coupled to an Esquire 3000+ (Bruker Daltonics, GmbH, Germany) mass spectrometer equipped with an electrospray ionization (ESI) source and ion-trap mass analyzer and operated by Esquire control and data analysis software. The column used was an Agilent Poroshell 120 RP-C 18 (4.6 by $150 \mathrm{~mm}, 2.7 \mu \mathrm{m})$. The sample separation was carried out using a linear gradient using $1 \%$ formic acid (A) and acetonitrile (B). The gradient started with $5 \% \mathrm{~B}, 25 \%$ at $30 \mathrm{~min}, 45 \% \mathrm{~B}$ at $45 \mathrm{~min}$, then $5 \%$ at $51 \mathrm{~min}$ and $5 \mathrm{~min}$ more for rebalancing. The flow rate was $0.5 \mathrm{~mL} / \mathrm{min}$ [21]. The diode-array detector was programmed at 280, 320, and $340 \mathrm{~nm}$. The operating conditions of the mass spectrometer were optimized to achieve maximum sensitivity values. The ESI system operated in negative mode to generate ions $[\mathrm{M}-\mathrm{H}]^{-}$under the following conditions: Desolvation temperature at $360^{\circ} \mathrm{C}$, vaporizer temperature at $400{ }^{\circ} \mathrm{C}$, drying gas (nitrogen), and nebulizer at $12 \mathrm{~L}$ per minute and 70 psi, respectively. The data was acquired as full exploration mass spectra from 50 to $1400 \mathrm{~m} / \mathrm{z}$ using $200 \mathrm{~ms}$ for the collection of the ions in the trap.

\subsection{Analysis of Fatty Acids}

The analysis of the fatty acid composition was carried out according to the International Olive Council method [18] with some modifications. Methylated esters were obtained from the mixture of $0.1 \mathrm{~g}$ of oil with $3 \mathrm{~mL}$ of hexane and $0.5 \mathrm{KOH}$ in methanol $0.2 \mathrm{~N}$. Fatty acid determination was performed using a gas chromatography GC (Agilent 7890B technology) equipped with a capillary column CP -sil 88 model CP6173 (50 m of length and $250 \mu \mathrm{m}$ of internal diameter). The film thickness was $250 \mu \mathrm{m}$, and the temperature was maintained at $225^{\circ} \mathrm{C}$ throughout the analysis time. The temperature of the injector was $230{ }^{\circ} \mathrm{C}$ while the detector temperature was $300^{\circ} \mathrm{C}$ and the oven temperature was set at $240^{\circ} \mathrm{C}$. Hydrogen was used as a carrier gas.

\subsection{Statistical Analysis}

Statistical significance was tested through a one-way ANOVA and Duncan test at $5 \%$ confidence level using SPSS statistical package (Version 12.0 for Window, SPSS Inc. Chicago, IL, 2003).

\section{Results}

\subsection{Quality Indices, Pigments, and Fatty Acid Composition of Olive Oil Samples}

Table 1 shows the variation of quality indices of olive oil samples from the three collecting dates before and after vertical centrifugation. After vertical centrifugation, all final-produced olive oil 
samples conformed to the norms fixed by the IOC for extra virgin olive oil class except for samples $4 \mathrm{~d} 1$ and $6 \mathrm{~d} 1$ (after vertical centrifugation).

Table 1. Quality indices of olive oil samples.

\begin{tabular}{|c|c|c|c|c|c|}
\hline \multicolumn{6}{|c|}{ 22-11-2017 } \\
\hline & Sample & FFAs & PV & $K_{232}$ & $\mathbf{K}_{270}$ \\
\hline \multirow{6}{*}{$\begin{array}{l}\text { before vertical } \\
\text { centrifugation }\end{array}$} & 1 & $0.4 \pm 0.8^{\mathrm{a}, \mathrm{b}}$ & $6 \pm 0.8^{a}$ & $1.90 \pm 0.02^{\mathrm{a}}$ & $0.1 \pm 0.1^{\mathrm{a}, \mathrm{b}}$ \\
\hline & 2 & $0.4 \pm 0.1^{\mathrm{a}, \mathrm{b}}$ & $\begin{array}{c}9.70 \pm 1.15 \\
\mathrm{a}, \mathrm{b}, \mathrm{c}, \mathrm{d}\end{array}$ & $1.9 \pm 0.4^{\mathrm{a}}$ & $0.07 \pm 0.04^{\mathrm{a}}$ \\
\hline & 3 & $0.5 \pm 0.1^{\mathrm{a}, \mathrm{b}}$ & $16.0 \pm 1.5^{\mathrm{e}}$ & $2.10 \pm 0.05^{\mathrm{a}}$ & $0.10 \pm 0.01^{\mathrm{a}, \mathrm{b}}$ \\
\hline & 4 & $0.40 \pm 0.02^{a, b}$ & $13 \pm 4.6^{\mathrm{d}}$ & $2.17 \pm 0.10^{\mathrm{a}}$ & $0.14 \pm 0.02^{c, d}$ \\
\hline & 5 & $0.4 \pm 0.1^{\mathrm{a}, \mathrm{b}}$ & $9 \pm 0.6^{a, b, c}$ & $2.3 \pm 0.3^{\mathrm{a}, \mathrm{b}}$ & $0.17 \pm 0.04^{b, c, d}$ \\
\hline & 6 & $0.40 \pm 0.01^{\mathrm{a}, \mathrm{b}}$ & $13.0 \pm 1.5^{\mathrm{d}}$ & $2.13 \pm 0.22^{a}$ & $0.15 \pm 0.03^{\mathrm{d}}$ \\
\hline \multirow{6}{*}{$\begin{array}{l}\text { after vertical } \\
\text { centrifugation }\end{array}$} & 1 & $0.43 \pm 0.06^{a, b}$ & $10 \pm 1 \mathrm{~b}, \mathrm{c}, \mathrm{d}$ & $2.12 \pm 0.1^{\mathrm{a}}$ & $0.12 \pm 0.02 \mathrm{a}, \mathrm{b}, \mathrm{c}$ \\
\hline & 2 & $0.30 \pm 0.01^{\mathrm{a}}$ & $6.7 \pm 2.3^{a, b}$ & $1.87 \pm 0.03^{\mathrm{a}}$ & $0.06 \pm 0.01^{\mathrm{a}}$ \\
\hline & 3 & $0.40 \pm 0.01^{\mathrm{a}, \mathrm{b}}$ & $10 \pm 1^{\mathrm{c}, \mathrm{d}}$ & $2.1 \pm 0.2^{\mathrm{a}}$ & $0.07 \pm 0.01^{\mathrm{a}}$ \\
\hline & 4 & $0.3 \pm 0.1^{\mathrm{a}}$ & $9.00 \pm 0.01^{a, b, c}$ & $2.6 \pm 0.5^{\mathrm{a}}$ & $0.18 \pm 0.10^{b, c, d}$ \\
\hline & 5 & $0.40 \pm 0.15^{\mathrm{a}, \mathrm{b}}$ & $10 \pm 2^{c d}$ & $2.3 \pm 0.1^{\mathrm{a}, \mathrm{b}}$ & $0.14 \pm 0.02^{c, d}$ \\
\hline & 6 & $0.3 \pm 0.1^{\mathrm{a}, \mathrm{b}}$ & $13.0 \pm 2.1^{\mathrm{d}}$ & $2.6 \pm 0.2^{b}$ & $0.20 \pm 0.04^{b, c, d}$ \\
\hline \multicolumn{6}{|c|}{ 19-12-2018 } \\
\hline & Sample & FFAs & PV & $\mathbf{K}_{232}$ & $\mathbf{K}_{270}$ \\
\hline \multirow{7}{*}{$\begin{array}{l}\text { before vertical } \\
\text { centrifugation }\end{array}$} & 1 & $0.5 \pm 0.0^{a, b, c}$ & $7.0 \pm 1.2^{\mathrm{a}, \mathrm{b}}$ & $1.9 \pm 0.1^{\mathrm{a}, \mathrm{b}}$ & $0.18 \pm 0.02^{b}$ \\
\hline & 2 & $1.2 \pm 0.1^{\mathrm{e}}$ & $9 \pm 2^{b, c, d}$ & $2.47 \pm 0.05^{\mathrm{d}}$ & $0.3 \pm 0.1^{\mathrm{c}}$ \\
\hline & 3 & $0.4 \pm 0.0^{\mathrm{a}}$ & $5.7 \pm 1.5^{\mathrm{a}}$ & $1.92 \pm 0.02^{\mathrm{a}, \mathrm{b}}$ & $0.18 \pm 0.01^{b}$ \\
\hline & 4 & $0.4 \pm 0.1^{\mathrm{a}, \mathrm{b}}$ & $9.0 \pm 1.2^{b, c}$ & $1.85 \pm 0.1^{\mathrm{a}, \mathrm{b}}$ & $0.18 \pm 0.01^{b}$ \\
\hline & 5 & $0.5 \pm 0.1^{\mathrm{a}, \mathrm{b}, \mathrm{c}}$ & $18.0 \pm 1.5^{\mathrm{g}}$ & $1.82 \pm 0.04^{\mathrm{a}, \mathrm{b}}$ & $0.180 \pm 0.004^{b}$ \\
\hline & 6 & $0.4 \pm 0.1^{\mathrm{a}, \mathrm{b}}$ & $7.0 \pm 1.2^{a, b}$ & $1.71 \pm 0.03^{\mathrm{a}}$ & $0.19 \pm 0.01^{b, c}$ \\
\hline & 7 & $0.5 \pm 0.2^{\mathrm{a}, \mathrm{b}}$ & $8.0 \pm 0.6^{a, b}$ & $2.07 \pm 0.05^{c}$ & $0.25 \pm 0.05^{c}$ \\
\hline \multirow{7}{*}{$\begin{array}{l}\text { after vertical } \\
\text { centrifugation }\end{array}$} & 1 & $0.6 \pm 0.2^{b, c}$ & $12 \pm 0.6^{\mathrm{e}, \mathrm{f}}$ & $1.9 \pm 0.1^{\mathrm{a}, \mathrm{b}}$ & $0.15 \pm 0.01^{\mathrm{a}, \mathrm{b}}$ \\
\hline & 2 & $0.9 \pm 0.1^{\mathrm{d}}$ & $13.7 \pm 1.5^{\mathrm{f}}$ & $2.3 \pm 0.1^{\mathrm{c}, \mathrm{d}}$ & $0.19 \pm 0.01^{b, c}$ \\
\hline & 3 & $0.5 \pm 0.1 \mathrm{ab}$ & $9 \pm 1^{b, c, d}$ & $1.99 \pm 0.05^{\mathrm{a}, \mathrm{b}}$ & $0.19 \pm 0.01^{b}$ \\
\hline & 4 & $0.7 \pm 0.1^{\mathrm{c}}$ & $12 \pm 0.6^{\mathrm{e}, \mathrm{f}}$ & $1.9 \pm 0.1^{\mathrm{a}, \mathrm{b}}$ & $0.11 \pm 0.01^{\mathrm{a}}$ \\
\hline & 5 & $0.6 \pm 0.1^{\mathrm{a}, \mathrm{b}, \mathrm{c}}$ & $10 \pm 0.6^{c, d, e}$ & $1.98 \pm 0.03^{a, b}$ & $0.14 \pm 0.03^{\mathrm{a}, \mathrm{b}}$ \\
\hline & 6 & $0.6 \pm 0.1^{\mathrm{a}, \mathrm{b}, \mathrm{c}}$ & $11 \pm 1^{\mathrm{d}, \mathrm{e}}$ & $1.88 \pm 0.01^{\mathrm{a}, \mathrm{b}}$ & $0.14 \pm 0.02^{\mathrm{a}, \mathrm{b}}$ \\
\hline & 7 & $0.5 \pm 0.1^{a, b, c}$ & $8 \pm 1^{b, c}$ & $1.9 \pm 0.2^{a, b}$ & $0.14 \pm 0.03^{a, b}$ \\
\hline \multicolumn{6}{|c|}{ 19-01-2018 } \\
\hline & Sample & FFAs & PV & $\mathbf{K}_{232}$ & $\mathbf{K}_{270}$ \\
\hline \multirow{4}{*}{$\begin{array}{l}\text { before vertical } \\
\text { centrifugation }\end{array}$} & 1 & $0.4 \pm 0.1^{\mathrm{a}, \mathrm{b}}$ & $6.0 \pm 1.5^{\mathrm{a}}$ & $1.8 \pm 0.1^{\mathrm{a}, \mathrm{b}}$ & $0.15 \pm 0.02^{a, b, c}$ \\
\hline & 2 & $0.4 \pm 0.1^{\mathrm{a}, \mathrm{b}}$ & $4.0 \pm 2.3^{\mathrm{a}}$ & $1.7 \pm 0.1^{\mathrm{a}}$ & $0.11 \pm 0.03^{\mathrm{a}}$ \\
\hline & 3 & $0.4 \pm 0.1^{\mathrm{a}, \mathrm{b}}$ & $11 \pm 2^{b}$ & $1.9 \pm 0.1 \mathrm{bc}$ & $0.16 \pm 0.01^{b, c}$ \\
\hline & 4 & $0.5 \pm 0.1^{\mathrm{a}}$ & $6.0 \pm 1.5^{\mathrm{a}}$ & $1.7 \pm 0.2^{\mathrm{a}}$ & $0.14 \pm 0.03 \mathrm{a}, \mathrm{b}$ \\
\hline \multirow{4}{*}{$\begin{array}{l}\text { after vertical } \\
\text { centrifugation }\end{array}$} & 1 & $0.4 \pm 0.1^{\mathrm{a}, \mathrm{b}}$ & $6.0 \pm 1.5^{\mathrm{a}}$ & $1.7 \pm 0.1^{\mathrm{a}, \mathrm{b}}$ & $0.14 \pm 0.01^{\mathrm{a}, \mathrm{b}}$ \\
\hline & 2 & $0.4 \pm 0.1^{\mathrm{a}}$ & $4 \pm 2.3^{\mathrm{a}}$ & $1.91 \pm 0.13^{b, c}$ & $0.16 \pm 0.03^{b, c}$ \\
\hline & 3 & $0.4 \pm 0^{\mathrm{a}}$ & $12.0 \pm 2.1^{b}$ & $1.9 \pm 0.1^{b, c}$ & $0.18 \pm 0.01^{\mathrm{c}}$ \\
\hline & 4 & $0.43 \pm 0.06^{a, b}$ & $6.0 \pm 1.5^{\mathrm{a}}$ & $2.02 \pm 0.04^{\mathrm{a}}$ & $0.17 \pm 0.01^{b, c}$ \\
\hline
\end{tabular}

(a-g) The different letters indicate a significant difference (Duncan's test, $p=0.05)$. Values are expressed as mean values and standard deviations over three repetitions. Free fatty acids (FFAs): Expressed as \% of C18:1 and peroxide value (PV): Expressed as meq $\mathrm{O}_{2} \mathrm{~kg}^{-1}$. 
Regarding pigments, a significant difference $(p<0.05)$ was observed between centrifuged and non-centrifuged oils. Interestingly, higher contents in chlorophylls and carotenoids were registered for centrifuged olive oil in comparison to non-centrifuged ones (Figure 1).

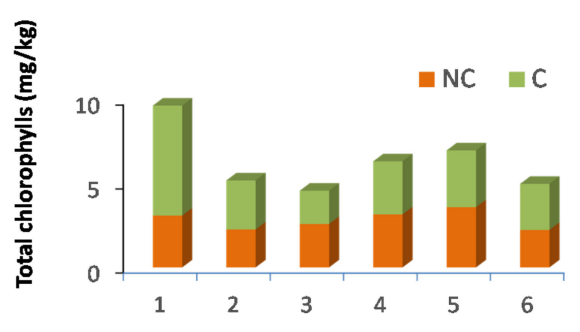

(a)

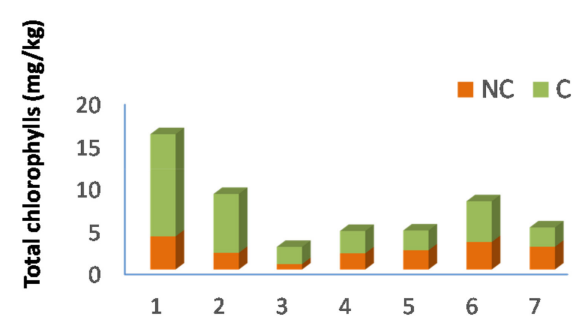

(c)

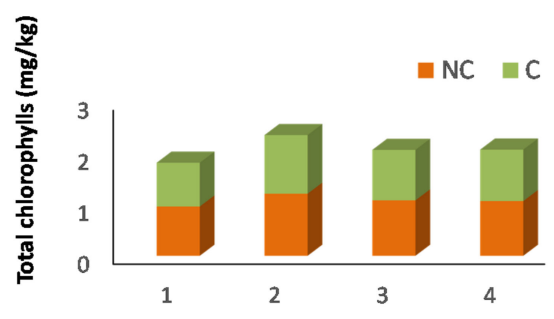

(e)

\section{2/11/2017}

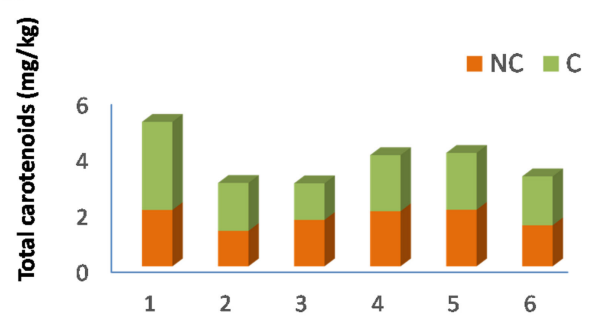

(b)

$19 / 12 / 2017$

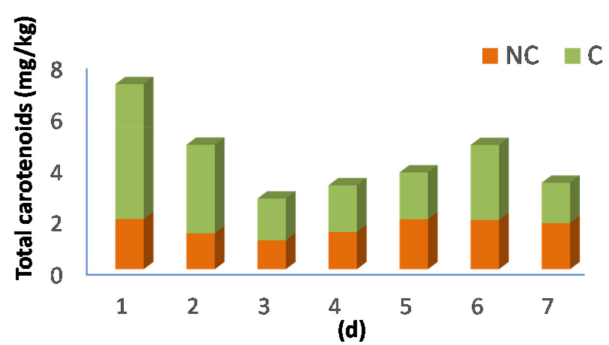

$19 / 01 / 2018$

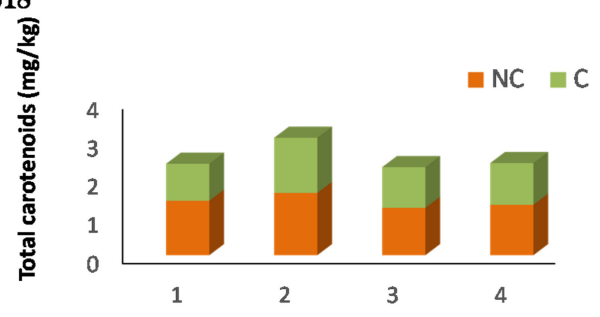

(f)

Figure 1. Pigment content variation according to vertical centrifugation and collecting period. (a) chlorophyll content variation in olive oils of 22/11/2017; (b) carotenoid content variation in olive oils collected at 22/11/2017; (c) chlorophyll content variation in olive oils at 19/12/2017; (d) carotenoid content variation in olive oils of 19/12/2017; (e) carotenoid content variation in olive oils collected at 19/01/2018; and (f) carotenoid content variation in olive oils of 19/01/2018. NC: Before vertical centrifugation, C: After vertical centrifugation.

The fatty acid composition of the studied samples is reported in Table 2. All obtained fatty acid percentages were conform to the International Olive Council standards for extra virgin olive oil class [18]. All samples were rich in monounsaturated acid (oleic acid) C18:1 with a maximum of 69.95\% (sample six at the second collecting date Table 2). 
Table 2. Fatty acid composition of olive oil samples (\% $\mathrm{m} / \mathrm{m}$ methyl esters).

\begin{tabular}{cccccccccccc}
\hline & Sample & C16:0 & C16:1 & C17:0 & C17:1 & C18:0 & C18:1 & C18:2 & C18:3 & C20:0 & C20:1 \\
\hline & 1 & 12.87 & 0.59 & 0.13 & 0.21 & 3.00 & 64.6 & 16.31 & - & 0.43 & 0.40 \\
& 2 & 12.11 & 0.52 & 0.19 & 0.35 & 2.81 & 65.62 & 15.17 & 0.75 & 0.38 & 0.42 \\
$\mathrm{~d} 1$ & 3 & 13.70 & 0.56 & 0.29 & 0.40 & 2.98 & 64.54 & 14.62 & 0.51 & 0.45 & 0.36 \\
& 4 & 12.71 & 0.52 & 0.11 & 0.13 & 3.00 & 64.32 & 16.97 & - & 0.45 & 0.36 \\
& 5 & 12.92 & - & 0.16 & - & 2.96 & 64.80 & 17.44 & 0.61 & 0.45 & 0.47 \\
& 6 & 12.05 & 0.39 & 0.47 & 0.21 & 2.92 & 62.88 & 19.17 & - & 0.46 & - \\
\hline & 1 & 13.07 & 0.51 & - & - & 3.12 & 64.94 & 16.48 & 0.49 & 0.41 & 0.45 \\
& 2 & 12.00 & 0.36 & - & - & 3.36 & 63.30 & 19.61 & 0.53 & 0.45 & 0.39 \\
& 3 & 10.69 & 0.27 & 0.16 & - & 3.77 & 65.13 & 18.30 & 0.52 & 0.45 & 0.36 \\
& 4 & 11.31 & 0.29 & - & - & 4.01 & 64.67 & 17.92 & 0.63 & 0.48 & 0.46 \\
& 5 & 16.44 & 1.28 & - & - & 3.16 & 63.16 & 12.67 & 1.00 & 0.49 & 0.40 \\
& 6 & 12.58 & 1.04 & 0.10 & - & 2.77 & 69.33 & 12.80 & 0.43 & 0.44 & 0.37 \\
& 7 & 16.47 & 0.87 & - & - & 3.18 & 62.45 & 13.86 & 0.41 & 0.46 & 0.46 \\
\hline & 1 & 11.03 & 0.46 & 0.14 & & 3.40 & 67.78 & 15.73 & 0.46 & 0.44 & 0.32 \\
& 2 & 11.69 & 0.24 & - & - & 3.44 & 65.20 & 16.67 & 0.47 & 0.46 & 0.42 \\
& 3 & 9.73 & 0.35 & 0.17 & - & 3.57 & 67.25 & 17.36 & 0.48 & 0.57 & 0.37 \\
& 4 & 11.74 & 0.15 & 0.44 & 0.05 & 3.11 & 65.88 & 16.99 & 0.53 & 0.57 & 0.38 \\
\hline EVOO (IOC [18]) & & $7.5-20$ & $3-3.5$ & - & - & $0.5-5$ & $55-83$ & $3.5-21$ & $\leq 1$ & $\leq 0.6$ & $\leq 0.5$ \\
\hline
\end{tabular}

d1: 22/11/2017, d2: 19/12/2017, and d3: 19/01/2018. Data are expressed by mean values \pm SD of three independent experiments. Values followed by same letters are not significantly different (Duncan's test, $p=0.05$ ).

\subsection{Total Polyphenols in the Extracts}

The distribution of total polyphenols (TP) in olive oil and by-product extracts is represented in (Table 3). A significant variations of TP contents between olive oil extracts as well as between by-products were observed. After vertical centrifugation, TP contents decreased for some olive oil samples and on the contrary increased in a part of olive oil samples. However, an apparent slight decrease was observed for ethyl acetate omww extracts after vertical centrifugation. Among by-products, ethyl acetate omww extracts showed the highest contents. We can say that ethyl acetate was more efficient than methanol in extracting omww polyphenols. For all studied samples, TP contents decreased slightly from olive paste to pomace extracts. 
Table 3. Total polyphenols in olive oil, paste, pomace, and omww extracts (expressed as \% GAE per extract).

\begin{tabular}{|c|c|c|c|c|c|c|c|c|c|}
\hline & Samples & Paste & Oonc & Ooc & Pomace & Omww nc EA & Omwwc EA & Omwwnc MeOH & Omwwc MeOH \\
\hline \multirow{6}{*}{ d1 } & 1 & $4.9 \pm 0.4^{\mathrm{a}}$ & $45.5 \pm 1.4^{b}$ & $36.8 \pm 1.7^{b}$ & $2.2 \pm 1.5^{a}$ & $13 \pm 4^{\mathrm{a}}$ & $13.6 \pm 4.4^{\mathrm{a}}$ & $2.7 \pm 0.4^{\mathrm{a}}$ & $8.7 \pm 2.9^{\mathrm{a}}$ \\
\hline & 2 & $1.2 \pm 1.2^{\mathrm{a}}$ & $35.6 \pm 1.8^{\mathrm{b}, \mathrm{c}}$ & $44.5 \pm 3.7^{c}$ & $2.7 \pm 2.0^{\mathrm{a}}$ & $11.3 \pm 3.4^{\mathrm{a}, \mathrm{b}}$ & $9.7 \pm 2.6^{a, b}$ & $3.2 \pm 0.8^{\mathrm{a}}$ & $3.9 \pm 1.7^{\mathrm{a}}$ \\
\hline & 3 & $2.5 \pm 0.4^{\mathrm{a}}$ & $44.8 \pm 2.5^{b}$ & $27.6 \pm 1.3^{b}$ & $2.1 \pm 0.8^{a}$ & $8.1 \pm 1.7^{\mathrm{a}}$ & $6.95 \pm 1.4^{\mathrm{a}}$ & $3.7 \pm 0.6^{\mathrm{a}}$ & $3.6 \pm 1.1^{\mathrm{a}}$ \\
\hline & 4 & $3.9 \pm 0.8^{a}$ & $36.9 \pm 1.6^{b}$ & $35.4 \pm 1.7^{b}$ & $1.6 \pm 0.3^{a}$ & $13.4 \pm 2.3^{\mathrm{a}}$ & $11.6 \pm 2.3^{\mathrm{a}}$ & $2.7 \pm 0.4^{\mathrm{a}}$ & $4.4 \pm 1.4^{\mathrm{a}}$ \\
\hline & 5 & $3.5 \pm 0.6^{\mathrm{a}}$ & $37 \pm 2^{b}$ & $35.5 \pm 1.7^{\mathrm{b}}$ & $1.3 \pm 0.2^{\mathrm{a}}$ & $24.6 \pm 6.3^{\mathrm{a}}$ & $19.4 \pm 4.5^{\mathrm{a}}$ & $6.0 \pm 3.1^{\mathrm{a}}$ & $5.5 \pm 1.1^{\mathrm{a}}$ \\
\hline & 6 & $2.3 \pm 0.2^{\mathrm{a}}$ & $43 \pm 2^{b}$ & $35.7 \pm 1.3^{b}$ & $0.7 \pm 0.1^{\mathrm{a}}$ & $20.7 \pm 3.5^{b, c}$ & $16.7 \pm 2.8^{\mathrm{a}, \mathrm{b}}$ & $5.2 \pm 1.6^{\mathrm{a}, \mathrm{b}}$ & $5 \pm 1.4^{\mathrm{a}, \mathrm{b}}$ \\
\hline \multirow{7}{*}{ d2 } & 1 & $2.8 \pm 0.4^{\mathrm{a}}$ & $37.7 \pm 2.0^{b}$ & $30.6 \pm 4.2^{b}$ & - & $10.1 \pm 5.1^{a}$ & $9.2 \pm 2.9^{a}$ & $2.9 \pm 0.2^{a}$ & $4.7 \pm 0.8^{a}$ \\
\hline & 2 & $3.5 \pm 0.3^{a}$ & $39.4 \pm 1.3^{b, c}$ & $49.8 \pm 4.5^{b}$ & - & $29.3 \pm 2.9^{a, b, c}$ & $22.5 \pm 2.1^{\mathrm{a}, \mathrm{b}, \mathrm{c}}$ & $7.5 \pm 1.8^{\mathrm{a}, \mathrm{b}}$ & $4.8 \pm 0.3^{\mathrm{a}}$ \\
\hline & 3 & $2.1 \pm 1.1^{\mathrm{a}}$ & $38.7 \pm 1.1^{b}$ & $69.4 \pm 2.8^{c}$ & - & - & $16.8 \pm 2.2^{\mathrm{a}, \mathrm{b}}$ & $5.3 \pm 1.8^{a}$ & - \\
\hline & 4 & $2.4 \pm 0.8^{a}$ & $31.5 \pm 1.1^{b}$ & $68.10 \pm 4.05^{c}$ & $2 \pm 1.1^{\mathrm{a}}$ & $12.7 \pm 2.6^{\mathrm{a}, \mathrm{b}}$ & $10 \pm 1.2^{a, b}$ & $7.1 \pm 2.3^{\mathrm{a}, \mathrm{b}}$ & $4.4 \pm 1.4^{\mathrm{a}, \mathrm{b}}$ \\
\hline & 5 & $2.2 \pm 0.9^{\mathrm{a}}$ & $34.21 \pm 1.6^{b}$ & $61.8 \pm 3.3^{c}$ & $1.5 \pm 0.2^{\mathrm{a}}$ & $11.5 \pm 1.4^{\mathrm{a}}$ & $10.4 \pm 2.3^{\mathrm{a}}$ & $3.8 \pm 1.4^{\mathrm{a}}$ & $5.44 \pm 0.4^{\mathrm{a}}$ \\
\hline & 6 & $1.02 \pm 1.03^{\mathrm{a}}$ & $34.3 \pm 9.1^{b}$ & $79.09 \pm 4.6^{c}$ & $0.06 \pm 0.64^{\mathrm{a}}$ & $15.1 \pm 5.2^{\mathrm{a}, \mathrm{b}}$ & $12.1 \pm 3.7^{\mathrm{a}, \mathrm{b}}$ & $7.7 \pm 2.4^{\mathrm{a}, \mathrm{b}}$ & $5.3 \pm 0.9^{a, b}$ \\
\hline & 7 & - & $31.1 \pm 9.8^{b, c}$ & $51.46 \pm 2.8^{c}$ & $0.3 \pm 1.5^{\mathrm{a}}$ & $16.4 \pm 6.3^{\mathrm{a}, \mathrm{b}}$ & - & $7.3 \pm 2.7^{\mathrm{a}, \mathrm{b}}$ & - \\
\hline \multirow{4}{*}{ d3 } & 1 & $6.6 \pm 0.5^{a}$ & $36 \pm 1.6^{b}$ & $49.3 \pm 3.1^{b}$ & $1.1 \pm 0.1^{a}$ & $10.4 \pm 2.9^{a}$ & $7.8 \pm 1.4^{a}$ & $7.0 \pm 1.4^{\mathrm{a}}$ & $3.6 \pm 0.6^{\mathrm{a}}$ \\
\hline & 2 & $2.6 \pm 0.3^{a}$ & $47.0 \pm 2.1^{b}$ & $45.1 \pm 3.2^{b}$ & $0.8 \pm 0.1^{\mathrm{a}}$ & $4.9 \pm 1.6^{\mathrm{a}}$ & $5.2 \pm 1.1^{\mathrm{a}}$ & $2.1 \pm 1.1^{\mathrm{a}}$ & $4.2 \pm 0.5^{\mathrm{a}}$ \\
\hline & 3 & $6 \pm 1^{\mathrm{a}}$ & $76.2 \pm 3.8^{c}$ & $48.4 \pm 1.9 .^{b}$ & $0.7 \pm 0.1^{\mathrm{a}}$ & $10.5 \pm 0.9^{\mathrm{a}}$ & $10 \pm 1^{\mathrm{a}}$ & $5.6 \pm 0.8^{a}$ & $7.3 \pm 1.4^{\mathrm{a}}$ \\
\hline & 4 & $2.2 \pm 0.4^{\mathrm{a}}$ & $39.4 \pm 1.3^{b}$ & $43.6 \pm 2.8^{b}$ & $0.4 \pm 0.1^{\mathrm{a}}$ & $14.6 \pm 1.7^{\mathrm{a}}$ & $12.1 \pm 1.2^{\mathrm{a}}$ & $5.6 \pm 2.5^{\mathrm{a}}$ & $5.1 \pm 0.8^{a}$ \\
\hline
\end{tabular}

d1: 22/11/2017, d2: 19/12/2017, d3:19/01/2018, oonc: Olive oil before vertical centrifugation, ooc: Olive oil after vertical centrifugation, omwwnc: Olive mill waste water before vertical centrifugation, omwwc: Olive mill waste water after vertical centrifugation, EA: Ethyl acetate extract, and MeOH: Methanolic extract. Data are expressed by mean values \pm SD of three independent experiments. ${ }^{(\mathrm{a}-\mathrm{c})}$ Values followed by the same letters are not significantly different (Duncan's test, $p=0.05$ ). 


\subsection{Evaluation of the Antioxidant Activity: TEAC Assay}

Among the studied samples, the TEAC test was performed on 22 extracts. The latter are the extracts from different matrices (products and by-products) from different collecting dates, and that showed the highest total polyphenol content. TEAC correlates positively to the polyphenol contents [22]. Figure 2 shows the TEAC variation in the extracts of the different samples under study.

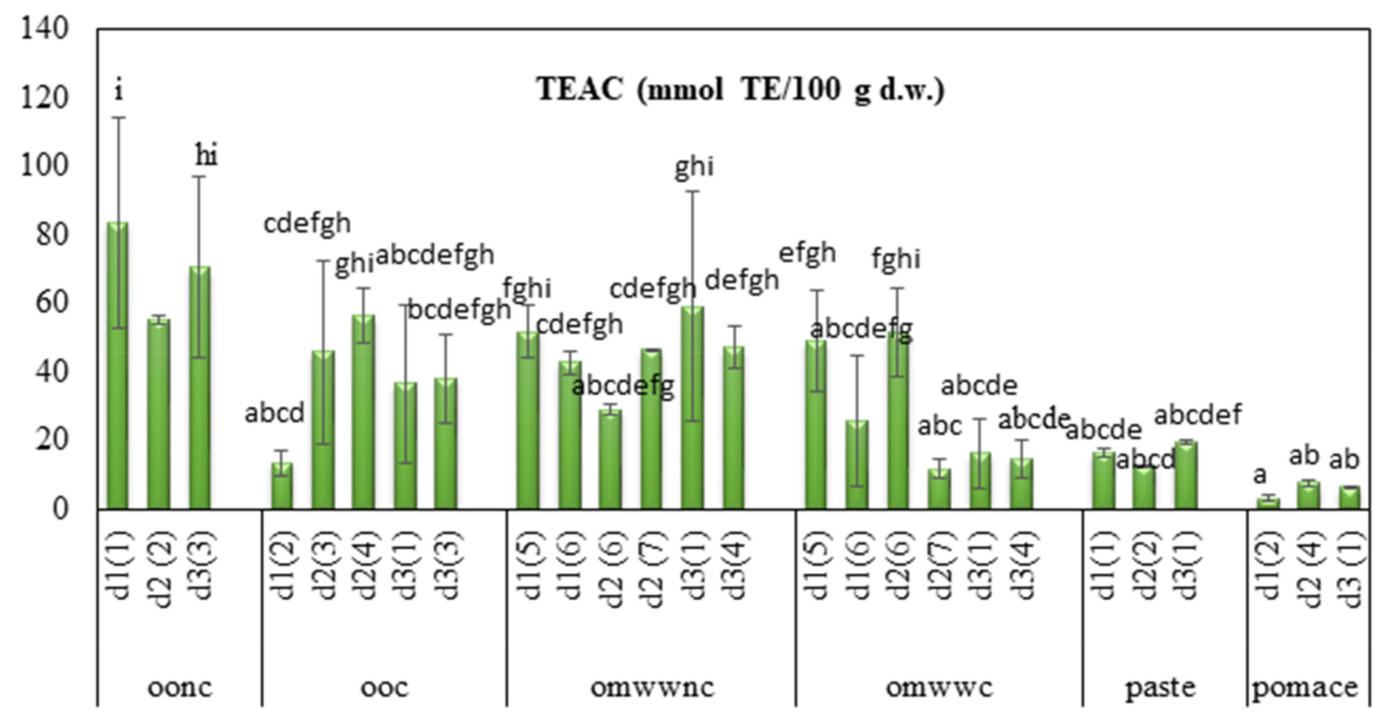

Figure 2. Trolox equivalent antioxidant capacity variation in olive oil, paste, and by-products. d1: 22/11/2017, d2: 19/12/2017, d3: 19/01/2018, oonc: Olive oil before vertical centrifugation, ooc: Olive oil after vertical centrifugation, omwwnc: Olive mill wastewater before vertical centrifugation, and omwwc: Olive mill wastewater after vertical centrifugation. (a-h) The different letters indicate a significant difference (Duncan's test, $p<0.05$ ) values are expressed as mean values and standard deviations over three repetitions.

\subsection{Phenolic Compound Analysis in Olive Paste, Olive Oil and by-Products}

The phenolic compounds were identified using high performance liquid chrmatography coupled to diode-array detector and electrospray ionization mass spectrometry (HPLC-DAD-ESI-MS/MS) analysis. The choice of samples to analyze in this part was based on TEAC activity in different matrices. The objective of this analysis was to assess the behavior of phenolic compounds in the different matrices during olive oil production process. Table 4 shows the identified compounds in olive oil, olive paste, and by-products. Nineteen phenolic compounds could be detected and determined based on available standard compounds, the MS and MS/MS fragments spectra, and the literature [23-30].

Regardless the collection date and vertical centrifugation process, as shown in Table 4 among samples, olive oil extracts showed the highest percentages in oleuropein aglycone (extracts 1, 9, 10, and 11) whereas, the rest of matrices extracts registered the highest percentages in secoiridoid derivative (extracts 2, 3, 5, 6, and 7) as well as in terms of hydroxyttyrosol hexoside dimer (extracts 2, 3, 6, and 7), acyclodihydroelenolic acid hexoside (extract 7), and G13 (extracts 4 and 5). Previously in literature [24] it was cited that most of these compounds are detected in drupe and paste.

In addition, other compounds were also determined such as stachyose which reached $13 \%$ in omww (7) extract. The compound (+)-1-Hydroxypinoresinol 1-O- $\beta$-D-glucopyranoside was present in omww extract (sample 6). This compounds was found in other researches [25] in wood, leaves, and stems of 'Chemlali' olive cultivar.

p-Coumaroyl-6-oleoside was present only in olive paste extracts (extracts two and three), reaching $14 \%$ in olive paste extract three. 
Table 4. Polar compounds determined in olive oil, paste, and by-products.

\begin{tabular}{|c|c|c|c|c|}
\hline Proposed Compound & M-H]- & MS/MS Fragments & Sample and Percentage & References \\
\hline Galloyl-HHDP-hexoside & 663 & - & $3(5.27 \%)$ & [23] \\
\hline Hydroxytyrosol hexoside dimer & 631 & $153 ; 315$ & $\begin{array}{c}2(15.00 \%), 3(11.09 \%), 6 \\
(17.48 \%), 7(13.85 \%)\end{array}$ & [24] \\
\hline Stachyose & 665 & & $4(2.64 \%), 7(13.85 \%)$ & [25] \\
\hline Secoirioid derivative & 815 & $407 ; 375 ; 313$ & $\begin{array}{c}2(63.66 \%), 3(39.98 \%), 5 \\
(50.49 \%), 6(35.20 \%), 7 \\
(37.85 \%)\end{array}$ & [26] \\
\hline $\begin{array}{c}\text { Acyclodihydroelenolic acid } \\
\text { hexoside }\end{array}$ & 407 & $389 ; 165$ & $4(12.75), 7(34.91 \%)$ & [25] \\
\hline Isonuezhenide & 685 & $\begin{array}{l}523 ; 453 ; 421 ; 299 ; \\
223\end{array}$ & $3(5.24 \%), 4(3.12 \%)$ & [27] \\
\hline Caffeoyl-6-oleoside & 551 & - & $2(6.61 \%), 3(6.75 \%), 6(6.56 \%)$ & [24] \\
\hline p-Coumaroyl-6-oleoside & 535 & 491 & $2(6.70 \%), 3(14.91 \%)$ & [24] \\
\hline $\begin{array}{l}\text { (+)-1-Hydroxypinoresinol } \\
\text { 1-O- } \beta \text {-D-glucopyranoside }\end{array}$ & 535 & - & $6(9.29 \%)$ & [25] \\
\hline $\begin{array}{c}\text { Elenolic acid dialdehyde linked } \\
\text { to hydroxytyrosol }\end{array}$ & 319 & 195 & $1(10.15 \%)$ & [28] \\
\hline G13 & 1071 & $\begin{array}{l}909 ; 837 ; 771 ; 685 ; \\
\quad 523 ; 385\end{array}$ & $\begin{array}{c}2(8.03 \%), 3(7.99 \%), 4(33.87 \%), \\
5(22.95 \%)\end{array}$ & [27] \\
\hline oleuropein aglycone & 377 & $275 ; 149$ & $\begin{array}{c}1(54.61 \%), 8(33.60 \%), 9 \\
(17.76 \%), 10(59.86 \%), 11 \\
(37.99 \%)\end{array}$ & [25] \\
\hline Forsythoside B & 755 & 447 & $8(12.30 \%)$ & [29] \\
\hline Leucosceptoside B & 781 & - & $6(9.63 \%)$ & [29] \\
\hline Secoisolariciresinol & 361 & - & $11(4.53 \%)$ & [30] \\
\hline Oleuropein hexoside & 701 & 539 & $1(6.06 \%)$ & [27] \\
\hline
\end{tabular}

Olive oil samples (1, 8, 9, 10, and 11), paste ( 2 and 3), pomace (4 and 5), and omww (6 and 7).

\section{Discussion}

No significant difference was observed among the quality parameters during the three collection dates, except for some samples such as $4 \mathrm{C}$ and $6 \mathrm{C}$ of 22/11/2018 that showed $\mathrm{k}_{232}$ values exceeding 2.5 $\left(K_{232}=2.6\right)$. Similarly, samples $2 \mathrm{NC}$ and $2 \mathrm{C}$ of 19/12/2017 represented a high percentage of free fatty acids $(1.2 \%$ and $0.9 \%$, respectively). Concerning the peroxide values, which evaluate the hydroperoxide content in olive oil and offer a measure of lipid oxidation, they ranged from $4 \mathrm{meqO}_{2} / \mathrm{kg}$ to $18 \mathrm{meqO}_{2} / \mathrm{kg}$ for all the studied samples. Peroxide values did not show an apparent variation according to the vertical centrifugation process (Table 1). Regarding free fatty acids, generally, no significant difference was noted between the centrifuged and non-centrifuged olive oils. A slight significant variation in samples collected in January, was found and especially for sample two free fatty acid content showed a decrease from $1.2 \%$ before centrifugation to $0.9 \%$ after centrifugation. Specific extinction values expressed a slight variation between centrifuged and non-centrifuged oils. Generally, variation obtained in terms of quality indices was observed mostly in samples according to centrifugation process more than the harvesting period. These findings are similar to those reported by other researchers [31] who assessed the variation of quality indices between olive oil obtained from processes of sedimentation and centrifugation with respect to raw olive oil obtained at the decanter exit.

The change in fatty acid composition can be related to the harvest time [32]. It was previously demonstrated that storage conditions, extraction process, and harvesting period might have an effect on the variation of the fatty acid content [32]. The slight variation in the fatty acid contents observed between analyzed samples seems to be also related to the cultivar, which is in accordance with previous researches [3].

According to the data of Table 3, it was found that omww polyphenol-extraction was more efficient with ethyl acetate than with methanol as an extractant solvent. omww comprises different bioactive phenolic compounds that have antimicrobial and phytotoxic effects. This composition is variable and depends on the cultivar, harvesting time, and extraction processes [33]. Its richness in water and nutriment gives to omww a significant value of fertilization. For this reason, it is advised to use this by-product for nutraceutical purposes due to its richness in nutritive components and its lower cost [34], since most of the phenolic compounds are lost lost during oil processing, ending up in wastes instead of oil [35]. 
Centrifuged and non-centrifuged olive oil samples presented good antioxidant capacities. However, a significant variation was observed in this parameter according to the matrix. The highest value was detected in olive oils followed by omww, paste, and pomace. Moreover, when extracted by ethyl acetate, the polar fraction of omww presented a higher value of TEAC compared to pomace extracts. A slight significant difference was registered between centrifuged and non-centrifuged olive oils and the same behavior was observed for omww (Figure 2). TEAC values for pomace extracts did not exceed $10 \mathrm{mmol}$ TE/100 g DW. Previous studies showed that the most significant influence of radical elimination was resulting from the presence of oleuropein aglycone dialdehyde (3,4-DHPEA-EDA) than other phenolic compounds [24].

The change in phenolic compounds is related to the activity of many enzymes that are released during pressing and malaxation steps [1]. It was previously demonstrated that all glycoside phenols are transformed to their aglycone forms, and the complex phenols are completely hydrolyzed to simple phenolics oleuropein, demethyloleuropein oleoside, and verbascoside [1]. In fact, polyphenol oxidase could be responsible for indirect oxidation of secoiridoids, and $\beta$-glucosidase could play a role in the production of phenol-aglycones such as the oleuropein aglycone and its isomers by hydrolysis of oleuropein as explained in literature [36]. The dialdehydic form of elenolic acid linked to hydroxytyrosol 3,4-DHPEA-EDA was detected in olive oil sample C4d2. The richness of olive oil in secoiridoid derivatives is an indicator of degradation pathways for the phenolic oleosides shown in the solid phases [36]. However, some compounds were identified in paste and by-products but not in olive oil such as hydroxytyrosol hexoside dimer, acyclodihydroelenolic acid hexoside, caffeoyl-6-oleoside, G13, and isonuezhenide (Table 4).

The presence of oleoside groups (caffeoyl-6-oleoside and p-coumaroyl-6-oleoside) in paste and by-products (Table 4) results probably from the degradation pathways of simple phenols [1]. The phenolic alcohol hydroxytyrosol hexoside dimer present in paste and by-products gave a base peak at m/z 631 with an MS/MS fragments at m/z 153 and m/z 315 which is in accordance with the results found elsewhere [24]. The presence of this compound in paste and by-products is mainly due to its strong hydrophilic nature and the activity of certain enzymes such as $\beta$-glucosidase during the malaxation process that is responsible for the hydrolysis of the glycosides to their respective aglycones [24].

The secoiridoids were present with their derivatives during the different steps of the extraction process specifically, in olive oil, paste, and by-products (Table 4). The transformation of the complex form of secoiridoid to the simple polyphenol form after malaxation is assured by the phenomenon of hydrolysis. So, the secoiridoids present in the paste or more precisely in fruit, are distributed between olive oil and by-product. However, it can be deduced that there is a significant loss of these major phenolic compounds in by-products. The secoiridoids are responsible for the good quality of virgin olive oil. Nevertheless, the extraction process of olive oil, such as the three-phase process, is responsible for the loss of a high amount of certain secoiridoids and their derivatives. On the other hand, galloyl hexahydroxydiphenoyl hexoside was detected in paste and a tetrasaccharide (stachyose) in by-products (Table 4). In our study, the distribution of phenolic compounds in paste, olive oil, and by-products differed significantly during the process of oil extraction.

According to previous works [37-41], the remarkable antioxidant capacity of olive oil, paste, and by-products may encourage their possible valorization. Further researches will be conducted to study in-depth the potential anti-cancer activity of selected extracts as novel cytotoxic agents.

\section{Conclusions}

The olive oil extraction process provides many valuable by-products thanks to their bioactive compounds. In this study, the matrices entailed in olive oil processing were screened for their quality indices and chemical composition (fatty acids, chlorophylls, carotenoids, and polyphenols). Different distribution of total polyphenols and values of TEAC antioxidant activity in olive oil, paste, and by-products (pomace and omww) extracts were highlighted in this study. Moreover, the distribution 
of individual phenolic compounds varied significantly according to the matrix. This variation is certainly linked to the transformation of initial phenolic compound in paste after malaxation due to the phenomenon of hydrolysis. Furthermore, we detected a considerable loss of secoiridoids glycosides and their derivatives in by-products. Generally, all samples from the three collecting dates shared similar behaviors for the studied parameters.

Author Contributions: Conceptualization, H.J. and A.T.; Methodology, H.J., M.L.-E. and F.J.Á.-M.; Software, H.J. and M.L.-E.; Validation, E.B.C. and A.T. and I.A.-R.; Formal Analysis, H.J., E.B.C. and A.T.; Investigation, H.J., M.L.-E. and F.J.Á.-M.; Resources, R.M. and E.B.C.; Data Curation, H.J. and F.J.Á.-M.; Writing-Original Draft Preparation, H.J.; writing-review and editing, H.N., I.A.-R. and A.T.; supervision, R.M. and E.B.C.; project administration, R.M. and E.B.C. All authors have read and agreed to the published version of the manuscript.

Funding: This research was funded by the Tunisian Ministry of Higher Education and by project RTI2018-096724-B-21 from the Spanish Ministry of Science, Innovation and Universities.

Conflicts of Interest: The authors declare no conflict of interest.

\section{References}

1. Talhaoui, N.; Gómez-Caravaca, A.M.; León, L.; De La Rosa, R.; Fernández-Gutiérrez, A.; Segura-Carretero, A. From olive fruits to olive Oil: Phenolic compound transfer in six different olive cultivars grown under the same agronomical conditions. Int. J. Mol. Sci. 2016, 17, 337. [CrossRef] [PubMed]

2. Servili, M.; Sordini, B.; Esposto, S.; Urbani, S.; Veneziani, G.; Di Maio, I.; Selvaggini, R.; Taticchi, A. Biological Activities of Phenolic Compounds of Extra Virgin Olive Oil. Antioxidants 2014, 1-23. [CrossRef] [PubMed]

3. Issaoui, M.; Dabbou, S.; Brahmi, F.; Hassine, K.B.; Ellouze, M.H.; Hammami, M. Effect of extraction systems and cultivar on the quality of virgin olive oils. Int. J. Food Sci. Technol. 2009, 44, 1713-1720. [CrossRef]

4. Chandra, M.; Sathiavelu, S. Waste management in the olive oil industry in the Mediterranean region by composting. Clean Technol. Environ. Policy 2009, 293-298. [CrossRef]

5. Dermeche, S.; Nadour, M.; Larroche, C.; Moulti-Mati, F.; Michaud, P. Olive mill wastes: Biochemical characterizations and valorization strategies. Process Biochem. 2013, 48, 1532-1552. [CrossRef]

6. Russo, M.; Bonaccorsi, I.L.; Cacciola, F.; Dugo, L.; De Gara, L.; Dugo, P.; Mondello, L. Distribution of bioactives in entire mill chain from the drupe to the oil and wastes. Nat. Prod. Res. 2020, 0, 1-6. [CrossRef] [PubMed]

7. Roig, A.; Cayuela, M.L.; Sa, M.A. An overview on olive mill wastes and their valorisation methods. Waste Manag. 2006, 26, 960-969. [CrossRef]

8. Azaizeh, H.; Halahlih, F.; Najami, N.; Brunner, D.; Faulstich, M.; Tafesh, A. Antioxidant activity of phenolic fractions in olive mill wastewater. Food Chem. 2012, 134, 2226-2234. [CrossRef]

9. Ciriminna, R.; Meneguzzo, F.; Fidalgo, A.; Ilharco, L.M.; Pagliaro, M. Extraction, benefits and valorization of olive polyphenols. Eur. J. Lipid Sci. Technol. 2016, 118, 503-511. [CrossRef]

10. Frankel, E.; Bakhouche, A.; Lozano-Sánchez, J.; Segura-Carretero, A.; Fernández-Gutiérrez, A. Literature review on production process to obtain extra virgin olive oil enriched in bioactive compounds. Potential use of byproducts as alternative sources of polyphenols. J. Agric. Food Chem. 2013, 61, 5179-5188. [CrossRef]

11. Ventura, G.; Calvano, C.D.; Abbattista, R.; Bianco, M.; De Ceglie, C.; Losito, I.; Palmisano, F.; Cataldi, T.R.I. Characterization of bioactive and nutraceutical compounds occurring in olive oil processing wastes. Rapid Commun. Mass Spectrom. 2019, 33, 1670-1681. [CrossRef] [PubMed]

12. El-Abbassi, A.; Kiai, H.; Hafidi, A. Phenolic profile and antioxidant activities of olive mill wastewater. Food Chem. 2012, 132, 406-412. [CrossRef] [PubMed]

13. Achmon, Y.; Fishman, A. The antioxidant hydroxytyrosol: Biotechnological production challenges and opportunities. Appl. Microbiol. Biotechnol. 2014. [CrossRef] [PubMed]

14. Ramos, P.; Santos, S.A.O.; Guerra, Â.R.; Guerreiro, O.; Felício, L.; Jerónimo, E.; Silvestre, A.J.D.; Neto, C.P.; Duarte, M. Valorization of olive mill residues: Antioxidant and breast cancer antiproliferative activities of hydroxytyrosol-rich extracts derived from olive oil by-products. Ind. Crops Prod. 2013, 46, 359-368. [CrossRef]

15. Nunzio, M.D.; Picone, G.; Pasini, F.; Caboni, F.; Gianotti, A.; Bordoni, A.; Capozzi, F. Olive oil industry by-products. Effects of a polyphenol-RICH extract on the metabolome and response to inflammation in cultured intestinal cell. Food Res. Int. 2018. [CrossRef] 
16. De Marco, E.; Savarese, M.; Paduano, A.; Sacchi, R. Characterization and fractionation of phenolic compounds extracted from olive oil mill wastewaters. Food Chem. 2007, 104, 858-867. [CrossRef]

17. Abaza, B.L.; Youssef, N.B.; Manai, H.; Haddada, F.M. Chétoui olive leaf extracts: Influence of the solvent type on phenolics and antioxidant activities. Grasas Aceites 2011, 62, 96-104. [CrossRef]

18. Norme Commerciale Applicable Aux Huiles D'Olive Et Aux Huiles De Grignons D'Olive. Cons. Oleic. Int. 2015, COI/T.15, 1-18.

19. De Príncipe, V.; Nunzio, M.D.; Toselli, M.; Verardo, V.; Caboni, F.; Bordoni, A. Counteraction of oxidative damage by pomegranate juice: Influence of the cultivar. J. Sci. Food Agric. 2013. [CrossRef]

20. Tomás-Menor, L.; Barrajón-Catalán, E.; Segura-Carretero, A.; Martí, N.; Saura, D.; Menéndez, J.A.; Joven, J.; Micol, V. The promiscuous and synergic molecular interaction of polyphenols in bactericidal activity: An opportunity to improve the performance of antibiotics? Phyther. Res. 2015, 29, 466-473. [CrossRef]

21. Talhaoui, N.; Gómez-Caravaca, A.M.; León, L.; De la Rosa, R.; Segura-Carretero, A.; Fernández-Gutiérrez, A. Determination of phenolic compounds of "Sikitita" olive leaves by HPLC-DAD-TOF-MS. Comparison with its parents "Arbequina" and "Picual" olive leaves. LWT_Food Sci. Technol. 2014, 58, 28-34. [CrossRef]

22. Barrajón-Catalán, E.; Fernández-Arroyo, S.; Saura, D.; Guillén, E.; Fernández-Gutiérrez, A.; Segura-Carretero, A.; Micol, V. Cistaceae aqueous extracts containing ellagitannins show antioxidant and antimicrobial capacity, and cytotoxic activity against human cancer cells. Food Chem. Toxicol. 2010, 48, 2273-2282. [CrossRef] [PubMed]

23. Fischer, U.A.; Carle, R.; Kammerer, D.R. Identification and quantification of phenolic compounds from pomegranate (Punica granatum L.) peel, mesocarp, aril and differently produced juices by HPLC-DAD-ESI/MSn. Food Chem. 2011, 127, 807-821. [CrossRef] [PubMed]

24. Kanakis, P.; Termentzi, A.; Michel, T.; Gikas, E.; Halabalaki, M.; Skaltsounis, A.L. From olive drupes to olive Oil An HPLC-orbitrap-based qualitative and quantitative exploration of olive key metabolites. Planta Med. 2013, 79, 1576-1587. [CrossRef] [PubMed]

25. Ammar, S.; Contreras, M.d.M.; Gargouri, B.; Segura-Carretero, A.; Bouaziz, M. RP-HPLC-DAD-ESI-QTOF-MS based metabolic profiling of the potential Olea europaea by-product "wood" and its comparison with leaf counterpart. Phytochem. Anal. 2017, 28, 217-229. [CrossRef] [PubMed]

26. Li, H.; Yao, W.; Liu, Q.; Xu, J.; Bao, B.; Shan, M.; Cao, Y.; Cheng, F.; Ding, A.; Zhang, L. Application of UHPLC-ESI-Q-TOF-MS to identify multiple constituents in processed products of the herbal medicine ligustri lucidi fructus. Molecules 2017, 22, 689. [CrossRef]

27. Shaoping, F.; Segura Carretero, A.; Arraez Roman, D. Tentative Characterization of Novel Phenolic Compounds in Extra Virgin Olive Oils by Rapid-Resolution Liquid Chromatography Coupled with Mass Spectrometry. J. Agric. Food Chem. 2009, 11140-11147. [CrossRef]

28. Kirmizibekmez, H.; Montoro, P.; Piacente, S.; Pizza, C.; Dönmez, A.; Çaliş, I. Identification by HPLC-PAD-MS and quantification by HPLC-PAD of phenylethanoid glycosides of five Phlomis species. Phytochem. Anal. 2005, 16, 1-6. [CrossRef] [PubMed]

29. Sanz, M.; De Simón, B.F.; Cadahía, E.; Esteruelas, E.; Muñoz, A.M.; Hernández, T.; Estrella, I.; Pinto, E. LC-DAD/ESI-MS/MS study of phenolic compounds in ash (Fraxinus excelsior L. and F. americana L.) heartwood. Effect of toasting intensity at cooperage. J. Mass Spectrom. 2012, 47, 905-918. [CrossRef]

30. Cardinali, A.; Pati, S.; Minervini, F.; D’Antuono, I.; Linsalata, V.; Lattanzio, V. Verbascoside, isoverbascoside, and their derivatives recovered from olive mill wastewater as possible food antioxidants. J. Agric. Food Chem. 2012, 60, 1822-1829. [CrossRef]

31. Altieri, G.; Di Renzo, G.C.; Genovese, F.; Tauriello, A.; D'Auria, M.; Racioppi, R.; Viggiani, L. Olive oil quality improvement using a natural sedimentation plant at industrial scale. Biosyst. Eng. 2014, 122, 99-114. [CrossRef]

32. Alowaiesh, B.; Singh, Z.; Fang, Z.; Kailis, S.G. Harvest time impacts the fatty acid compositions, phenolic compounds and sensory attributes of Frantoio and Manzanilla olive oil. Sci. Hortic. 2018, 234, 74-80. [CrossRef]

33. Barbera, A.C.; Maucieri, C.; Cavallaro, V.; Ioppolo, A.; Spagna, G. Effects of spreading olive mill wastewater on soil properties and crops, a review. Agric. Water Manag. 2013, 119, 43-53. [CrossRef]

34. Sellami, F.; Jarboui, R.; Hachicha, S.; Medhioub, K.; Ammar, E. Co-composting of oil exhausted olive-cake, poultry manure and industrial residues of agro-food activity for soil amendment. Bioresour. Technol. 2008, 99, 1177-1188. [CrossRef] [PubMed] 
35. Jerman Klen, T.; Mozetič Vodopivec, B. The fate of olive fruit phenols during commercial olive oil processing: Traditional press versus continuous two- and three-phase centrifuge. LWT Food Sci. Technol. 2012, 49, 267-274. [CrossRef]

36. Artajo, L.S.; Romero, M.P.; Suárez, M.; Motilva, M.J. Partition of phenolic compounds during the virgin olive oil industrial extraction process. Eur. Food Res. Technol. 2007, 225, 617-625. [CrossRef]

37. Reboredo-Rodríguez, P.; González-Barreiro, C.; Cancho-Grande, B.; Forbes-Hernández, T.Y.; Gasparrini, M.; Afrin, S.; Cianciosi, D.; Carrasco-Pancorbo, A.; Simal-Gándara, J.; Giampieri, F.; et al. Characterization of Phenolic Extracts from BRAVA Extra Virgin Olive Oils and Their Cytotoxic Effects on MCF-7 Breast Cancer Cells. Food Chem. Toxicol. 2018, 119, 73-85. [CrossRef]

38. Barrajón-Catalán, E.; Taamalli, A.; Quirantes-Piné, R.; Roldan-Segura, C.; Arráez-Román, D.; Segura-Carretero, A.; Micol, V.; Zarrouk, M. Differential metabolomic analysis of the potential antiproliferative mechanism of olive leaf extract on the JIMT-1 breast cancer cell line. J. Pharm. Biomed. Anal. 2015, 105, 156-162. [CrossRef]

39. Bouallagui, Z.; Han, J.; Isoda, H.; Sayadi, S. Hydroxytyrosol rich extract from olive leaves modulates cell cycle progression in MCF-7 human breast cancer cells. Food Chem. Toxicol. 2011, 49, 179-184. [CrossRef]

40. Taamalli, A.; Arráez-Román, D.; Barrajón-Catalán, E.; Ruiz-Torres, V.; Pérez-Sánchez, A.; Herrero, M.; Ibañez, E.; Micol, V.; Zarrouk, M.; Segura-Carretero, A.; et al. Use of advanced techniques for the extraction of phenolic compounds from Tunisian olive leaves: Phenolic composition and cytotoxicity against human breast cancer cells. Food Chem. Toxicol. 2012, 50, 1817-1825. [CrossRef]

41. Elamin, M.H.; Daghestani, M.H.; Omer, S.A.; Elobeid, M.A.; Virk, P.; Al-Olayan, E.M.; Hassan, Z.K.; Mohammed, O.B.; Aboussekhra, A. Olive oil oleuropein has anti-breast cancer properties with higher efficiency on ER-negative cells. Food Chem. Toxicol. 2013, 53, 310-316. [CrossRef] [PubMed]

(C) 2020 by the authors. Licensee MDPI, Basel, Switzerland. This article is an open access article distributed under the terms and conditions of the Creative Commons Attribution (CC BY) license (http://creativecommons.org/licenses/by/4.0/). 